\section{Anaesthesia for the CKD patient}

\section{Laura Baxter}

The kidneys are responsible for filtering the blood and controlling how much fluid is reabsorbed back into the circulatory system. To do this effectively they have to receive $20 \%$ of cardiac output. Blood flow to the kidneys is controlled by autoregulation which is effective at mean arterial blood pressures of between $60 \mathrm{mmHg}$ and $150 \mathrm{mmHg}$

Patients with renal disease have a reduced ability to compensate during stressful situations such as anaesthesia and surgery. If these patients are not managed carefully during the pre-, peri- and postanaesthesic periods their renal disease can progress to acute renal failure. It is therefore vitally important that intravascular volume, cardiac output, blood pressure and oxygenation to the kidneys are maintained during a patient's anaesthetic period.

Preanaesthetic patient assessment is important to establish a baseline for these patients. Cats in chronic renal failure are commonly hypertensive, so it is important to establish the severity before they are anaesthetized. It is also important to identify those that require stabilization before undergoing anaesthesia, as a minimum this should incorporate fluid therapy. Assessment can also aid in the choice of drugs that will be used. Drugs that are excreted unchanged or have active metabolites should be avoided in renal disease patients as their duration of action and effects may be unpredictable. Drugs that produce hypotension should also be avoided or used sparingly as they will effect autoregulation and blood flow to the kidneys. A balanced anaesthetic technique will allow lower doses of drugs to be used in an effort to reduce undesirable side effects.

Renal disease patents should be carefully monitored whilst anaesthetized so that problems can be quickly identified and acted upon, in particular hypotension. Consideration should also be given to the patient's body condition as renal disease patients can be thin and, as such, prone to hypothermia resulting in a prolonged recovery from anaesthesia. In the postoperative period patients should be monitored to ensure they have adequate urine output. They may also require blood tests and urinalysis to assess for worsening of their renal disease.

\section{KEY LEARNING OBJECTIVES}

- Understand the basic pathophysiology of renal disease

- Identify the needs specific to renal disease patients in the pre-, peri- and postanaesthetic periods

- Understand which drugs are appropriate for renal disease patients and those to be avoided

\section{MULTIPLE CHOICE QUESTIONS}

1. What is the first thing that can be done to manage hypotension in the anaesthetized renal disease patient?
(A) Turn down the volatile agent
(B) Turn up the intravenous fluid rate
(C) Administer a bolus of fluids
(D) Administer a sympathomimetic drug

2. Why should ketamine be avoided in renal disease patients?
(A) It is excreted unchanged in cats and as an active metabolite in dogs
(B) It increases cardiac output
(C) Onset of action is relatively slow
(D) It causes discomfort on intramuscular injection

3. At what level should mean arterial pressure be maintained in anaesthetized cats in chronic renal failure with hypertension?
(A) $>70-80 \mathrm{mmHg}$
(B) $60-70 \mathrm{mmHg}$
(C) $40-50 \mathrm{mmHg}$
(D) $100-120 \mathrm{mmHg}$

\section{Anaesthesia for the cardiac patient}

\section{Laura Baxter}

It is not uncommon for patients that present for routine procedures requiring anaesthesia or sedation to be suffering from cardiac disease. These patients may be well controlled on a drug regime, unstable or not even diagnosed. Patients with acquired heart conditions can also present in crisis due to progression of their disease and subsequent development of other conditions, for example, the Cavalier King Charles Spaniel with degenerative mitral valve disease presenting with dyspnoea due to pulmonary oedema.

There are generalized aims when anaesthetizing these patients to try and allow the cardiovascular system to operate normally:

- Maintain that patient's normal heart rate - avoid stress or pain which may increase rate, avoid vagal stimulation which may decrease rate

- Maintain myocardial function

- Maintain cardiac output

- Maintain arterial blood pressure within normal ranges

- Avoid increasing myocardial function and, as such, myocardial oxygen demand

- Maintain oxygenation - maintain good ventilation, provide oxygen as necessary. 
Every effort should be made to reduce stress in these patients as an increase in heart rate can have a major impact on their cardiovascular function. A thorough preanaesthetic assessment should be carried out to identify any cardiovascular abnormalities which may require further investigation before anaesthesia. Before any drugs are given everything should be prepared and ready to anaesthetize the patient; this includes availability of emergency drugs with doses calculated. A balanced anaesthetic technique will allow lower drug doses to be used, reducing undesirable cardiovascular side effects.

Induction agents often have significant effects on the cardiovascular system, making induction one of the most critical times for cardiac patients. If tolerated, monitoring equipment should be attached before induction to help identify adverse events. Pre-oxygenation, if tolerated, will help to maintain oxygenation should hypoventilation occur during induction. Patients should be monitored closely during anaesthesia with problems being identified and acted upon quickly. They should be recovered in a calm and stress-free environment and monitored until their parameters are within normal ranges. Analgesia will be important for these patients as stimulation of the sympathetic nervous system can result in increased heart rate.

Some common acquired heart conditions and their anaesthetic considerations include:

- Degenerative mitral valve disease:

- Maintain heart rate (avoid bradycardia)

- Maintain preload by maintaining venous return

- Maintain contractility

- Avoid increasing afterload by reducing systemic vascular resistance

- Hypertrophic cardiomyopathy:

- Avoid stress!

- Avoid bradycardia and tachycardia

- Maintain intravascular volume

- Dilated cardiomyopathy:

- Maintain cardiac output by maintaining heart rate and preload

- Monitor closely for arrhythmias
Pericardial effusion:

- Maintain heart rate

- Maintain preload by maintaining venous return.

\section{KEY LEARNING OBJECTIVES}

- Identify the aims when anaesthetizing cardiac patients

- Understand the generalized anaesthetic protocol for cardiac patients

- Identify some key points for anaesthetizing patients with common acquired heart conditions, for example, cardiomyopathies, valve disease and pericardial effusion

\section{MULTIPLE CHOICE QUESTIONS}

1. Why is stress a particular concern for cardiac patients?

(A) It stimulates the sympathetic nervous system resulting in an increase in heart rate which may not be tolerated by cardiac patients

(B) It makes patients difficult to handle

(C) It decreases drug doses

(D) It deceases oxygen demand

2. Why is anaesthesia preferable to sedation for cardiac patients?

(A) It allows endotracheal intubation and the reliable delivery of oxygen

(B) It provides better immobility

(C) It is quicker

(D) It requires less drugs

3. What is it important to maintain in anaesthetized cardiac patients?
(A) Cardiac output
(B) Hypotension
(C) Tachycardia
(D) Bradycardia 